\title{
Review
}

\section{The Underutilization of Lifestyle Modifications in Primary Care Medicine}

\author{
Jean-Marc Lucas ${ }^{1,2^{*}}$, Karl F. Kozlowski $^{3}$ \\ ${ }^{1}$ Former student, Canisius College, Buffalo, United States. \\ ${ }^{2}$ Student, SUNY Buffalo, Buffalo, United States. \\ ${ }^{3}$ Department of Kinesiology, Canisius College, Buffalo, United States.
}

\section{Article Information \\ History: \\ Received: October 17, 2018 \\ Accepted: January 11, 2019 \\ Published: February 15, 2019}

\section{Keywords:}

Chronic disease

Exercise

Lifestyle medicine

Nutrition

Obesity

\begin{abstract}
Chronic disease accounts for the majority of deaths in the United States and is often attributed to obesity. A sedentary lifestyle and poor nutrition are primary contributing factors to the development of obesity and thus chronic disease. Primary care providers are optimally positioned to prescribe exercise and nutrition (lifestyle medicine) as a treatment for chronic disease. Unfortunately, this opportunity seems to be regularly lost. Primary care providers often rely too heavily on weight loss pharmaceuticals and bariatric surgeries to treat obesity. This treatment approach however also does little to prevent and treat the accumulation of chronic diseases. The purpose of this review was to evaluate the efficacy of conventional medical weight loss treatments and determine why primary care providers may not prescribe exercise and nutrition more frequently. Our findings suggest that some primary care providers may be uncomfortable prescribing lifestyle medicine as they receive little formal education in this field. In conclusion, prescription of exercise and nutrition by primary care providers may elicit greater longterm weight loss than current medical weight management practices. Medical management is most likely effective when combined with lifestyle medicine. We propose that primary care providers be better trained in lifestyle medicine through their formal and clinical education. Rates of chronic disease accumulation may potentially decrease if providers prescribe lifestyle medical treatments more frequently.
\end{abstract}

\section{INTRODUCTION}

$\mathrm{C}$ hronic disease currently accounts for the majority of deaths in the United States [1]. Although modern medical treatments exist to combat chronic disease, they are mostly ineffective. The Center for Disease Control (CDC) has identified that over $71 \%$ of the U.S. population is overweight [2]. Obesity often results in the accumulation of chronic diseases such as diabetes and cardiovascular disease, which drastically decreases quality of life and longevity. The U.S. Department of Health and Human Services suggested that over $31 \%$ of adults in the U.S. are living with at least two or more chronic conditions in 2014 [3]. The top two chronic diseases in 2016, cardiovascular disease and cancer,

*Correspondence: Jean-Marc Lucas, Department of Kinesiology, Canisius College, 8311 Silver Fox Ct., Buffalo, United States.

E-mail address: jlucas2@buffalo.edu accounted for $45 \%$ of the total deaths that year [4].

High infectious disease mortality rates have decreased over time due to the advent of modern medicine. As such, people have been living long enough to accumulate a myriad of chronic diseases. In turn, chronic disease has become the leading cause of death in developed nations [5]. Chronic disease is generally understood as a class of noncommunicable diseases that progress at a slow rate and persist for extended periods of time [6]. Among the top chronic diseases today, the World Health Organization (WHO) recognizes cardiovascular disease, diabetes, and cancer as having a negative impact internationally [6]. Seven of the top 10 leading causes of death in the U.S. are from chronic disease (cardiovascular disease, some cancers, chronic lower respiratory disease, cerebrovascular disease, Alzheimer's disease, diabetes, kidney disease) [4].

One of the root causes behind the recent rise of chronic disease lies buried in the modern lifestyle. A sedentary lifestyle can be a major contributing factor to the onset of chronic disease. Accelerometer data indicated that adults terms of the Creative Commons Attribution License (http://creativecommons.org/licenses/by/4.0/), which permits unrestricted use, distribution, and reproduction in any medium, provided the original work is properly cited. 
are sedentary for almost $60 \%$ of their waking hours each day [7]. Prolonged periods of inactivity increase biomarkers linked to chronic diseases such as cardiovascular disease, type 2 diabetes, and metabolic syndrome [8]. A sedentary lifestyle can increase all-cause mortality by up to 93\% [9]. A sedentary lifestyle also results in decreased daily net caloric expenditure, which heightens the risk of obesity. Genetic influences may also play a role in the onset of and susceptibility to chronic disease and obesity [10].

\section{Obesity as a Primary Source of Chronic Disease}

Since obesity is a large contributor to the accumulation and progression of chronic diseases, the treatment of obesity can decrease symptoms directly associated with excess weight gain, while simultaneously treating and preventing chronic disease. The CDC reported that about $40 \%$ of the adult U.S. population was obese in 2015 [11]. This indicates that current methods of preventing and treating excess weight gain are largely unsuccessful.

Clinical measurement of weight most often utilizes body mass index (BMI), the ratio of a person's weight in kilograms to the square of their height in meters. BMI is then compared against national averages to determine weight status (BMI of $25 \mathrm{~kg} / \mathrm{m} 2$ to $29.9 \mathrm{~kg} / \mathrm{m} 2$ is overweight, $30 \mathrm{~kg} / \mathrm{m} 2$ to $39.9 \mathrm{~kg} / \mathrm{m} 2$ is obese, and $40 \mathrm{~kg} / \mathrm{m} 2$ or greater is extreme obesity) [2]. Health care providers use BMI to quickly estimate chronic disease risk. The WHO warns that a higher BMI is correlated with greater risk for disease accumulation [6]. $\mathrm{BMI}$ is useful for most of the non-exercising population as a general health indicator, however it is an incomplete method for determining overweight status and morbidity risk due to its inability to distinguish between different types of body tissue.

A current review of clinical obesity management suggests that physicians should be first recommending lifestyle modifications, followed by pharmaceutical prescription, and lastly bariatric (weight loss) surgery to their obese patients [12]. According to Erlandson, et al., lifestyle recommendations should include increasing physical activity, making dietary modifications, and participating in behavioral therapies. Prescription weight loss pharmaceuticals, or pills, are recommended for patients with a BMI of $30 \mathrm{~kg} / \mathrm{m} 2$ or higher or $27 \mathrm{~kg} / \mathrm{m} 2$ or above with obesity related comorbidities. Bariatric surgery, or weight loss surgery, is recommended for patients with a BMI above $40 \mathrm{~kg} / \mathrm{m} 2$ [12].

\section{Pharmaceutical Induced Weight Loss}

Pharmaceuticals have been shown to induce statistically significant weight loss [13]. Despite reported pharmacotherapy success, several factors impact the overall usefulness of drug-therapy, including strict eligibility requirements, treatment corequisites, side effects, actual risk reduction, and long-term effectiveness.

Clinically obese individuals meeting BMI standards $(\geq$ $30 \mathrm{~kg} / \mathrm{m} 2$ or $\geq 27 \mathrm{~kg} / \mathrm{m} 2$ with obesity related comorbidities) are cleared for prescription pharmaceutical intervention [12]. While obese individuals are $81 \%$ more likely to develop coronary heart disease (CHD); simply being overweight (BMI $25-29.9 \mathrm{~kg} / \mathrm{m} 2$ ) is associated with a $32 \%$ increased risk of developing CHD relative to individuals with a normal BMI range of $18.5-24.9 \mathrm{~kg} / \mathrm{m} 2$ [14]. Despite this risk, weight loss pharmaceuticals are reserved for obese individuals, those who will maximize the risk-reward ratio of treatment. Approximately 2.7 million obese patients were using some form of anti-obesity medication in 2011, which accounts for not even $3 \%$ of all of the obese adults in the U.S. [15].

Most drugs have between 10 and 100 possible side effects or adverse drug reactions [16]. Typical side effects common to weight loss drugs are constipation, nausea and vomiting, diarrhea, and incontinence [17]. Some of the worst side effects include liver toxicity, cardiotoxicity, and drug abuse [18]. Side effects can also include an increase in blood pressure and heart rate which are directly correlated with increased cardiovascular disease morbidity and mortality [19].

Patients regain weight after pharmaceutical weight loss treatment ceases without parallel lifestyle changes [20]. Implementing pharmacotherapy following lifestyle modifications may be the most credible solution to optimize drug-aided weight loss [21]. Despite evidence of metabolic risk factor improvement, obesity medications have not been shown to subsequently reduce cardiovascular morbidity or mortality [13]. Lifestyle changes are thus concurrently necessary to reduce chronic disease risk.

In an effort to combat regaining weight, pharmaceuticals have been designed for both short-term ( $\leq 12$ weeks) and long-term use (as long as desired) [13]. The FDA has approved three weight loss drugs for long-term use as of 2014, which includes orlistat, lorcaserin, and phentermine-topiramate [13]. Yet these long-term medications do not work indefinitely. After a period of about 54-67 weeks, these medications reach their maximal efficacy point and weight regain is expected [20]. Weight regain typically ranges from one to three pounds every year after the maximal efficacy point has occurred [20].

\section{Surgically Assisted Weight Loss}

Bariatric surgery either limits the amount of food the stomach can hold (restrictive), reduces nutrient absorption efficiency (malabsorptive), or both (mixed) [22]. Bariatric surgeries commonly reduce some metabolic risk factors for chronic disease accumulation, such as blood glucose levels [22]. Bariatric surgery may be performed on the most at-risk patients as a life-saving procedure in order to prevent an anticipated chronic disease related death. Weight loss from bariatric surgery maximizes at two to four years post-surgery with a BMI reduction of up to $12-17 \mathrm{~kg} / \mathrm{m} 2$, or the equivalent of an $80 \%$ reduction in body weight [23]. In $2016 ; 216,000$ bariatric surgeries were performed in the U.S., a $36 \%$ increase from 2011 [24]. Despite this increase, this accounts for less than $1 \%$ of all morbidly obese individuals in the U.S. 
A patient must have a BMI of at least $40 \mathrm{~kg} / \mathrm{m} 2$ or $35 \mathrm{~kg} /$ $\mathrm{m} 2$ with another obesity related illness to be considered for bariatric surgery [22]. Prior to weight loss surgery, psychological health and dietary tendencies must be evaluated [25]. Preoperative patients must also show continued weight loss in order to be eligible for bariatric surgery. In order to optimize surgical results, physicians will evaluate a patient's willingness and motivation for surgery and their propensity for sustained lifestyle modifications post surgical treatment [25].

Regardless of procedure complexity, all bariatric surgeries pose risks of complications up to and including death [26]. The complication rate for bariatric surgery ranges from $10 \%-17 \%$ [23]. Approximately $7 \%$ of all bariatric surgery patients will require additional surgery beyond the initial bariatric procedure. Bariatric postoperative death rates are $6 \%$ in the five years after surgery [27]. Older patients $(\geq 55$ years) have the greatest risk of postoperative mortality $(62 \%$ in men, $25 \%$ in women). Common causes of death in the nine years after a bariatric surgery, are coronary heart disease (19\% of deaths), sepsis (14\%), pulmonary embolism (11\%), therapeutic complications (11\%), and cancer (10\%) [27].

Bariatric surgeries certainly yield short-term weight loss, but most procedures eventually lead to some weight being regained. In order to prevent as much weight regain as possible, patients must sustain healthy lifestyle modifications post surgery [28]. About 25\%-30\% of gross weight loss following bariatric surgery is typically regained over the subsequent several years [29]. Despite some regained weight, obese patients undergoing bariatric surgery are less likely to be diagnosed with diabetes or experience adverse cardiovascular events for over 10 years post surgery when compared with nonsurgical obese patients. Bariatric surgeries however do not provide a long term reduction of a patient's cholesterol levels or hypertension which indicates a continued risk for the development of chronic disease [29]. While bariatric surgery may help reduce the onset of some chronic disease, widespread application to all obese individuals is not viable due to the prequalification requirements of the bariatric patient and the overall risk to reward ratio of the surgery.

\section{Physical Activity}

Physical activity is presumed to help tilt the caloric balance toward a caloric deficit and thus elicit weight loss [30]. It is likely that aerobic exercise yields the greatest benefits for overweight people by providing the greatest increases in cardiorespiratory fitness and short-term weight loss [31]. Aerobic exercise increases metabolic rate (and thus energy expenditure) over the 24 hours post-exercise [32]. The primary benefits from physical activity reside beyond weight loss, and include influence over several metabolic factors and organs [33]. These benefits include increases in bone density, support for and creation of new vascular tissue, increases in fat metabolism, insulin sensitivity, memory and brain function, and decreases in systemic inflammation
[33]. For example, physically active obese men and women were $88 \%$ less likely to be insulin resistant than their inactive counterparts [34].

Until recently, the heart was believed to be incapable of generating new contractile cardiomyocyte cells [35]. Yet, several recent studies have theorized that exercise stimulates local cardiomyocyte stem cells and cardiomyocyte progenitor cells to increase proliferation and specialization to a greater extent [35]. This finding is critical to patients who have experienced some form of ischemic heart failure resulting in cardiac cell death, such as myocardial infarction. Additionally, aerobically fit men and women were $47 \%$ and $70 \%$ less likely to incur cardiovascular disease, respectively and are 50\% less likely to incur all-cause mortality [36].

\section{Nutrition}

The WHO states that $30 \%$ of all cancers, $80 \%$ of cardiovascular diseases, and $90 \%$ of type- 2 diabetes cases could be prevented in part by consuming a nutritious and balanced diet [37]. While approximately $75 \%$ of the U.S. population insufficiently consumes vegetables, fruits, dairy, and healthy fats, the U.S. Department of Agriculture (USDA) states that a healthy diet including plenty of vegetables and fruits can lower the risk for developing obesity, cardiovascular disease, some types of cancers, and type-2 diabetes [38]. Fruits and vegetables are important for their low energy density (low amount of calories per serving) and high phytochemical, vitamin, and mineral concentrations [39].

Oils are a type of unsaturated fat typically viewed as a "healthy" fat, relative to other "unhealthy" or saturated fats, such as butter or lard. Saturated fats have traditionally been associated with increased morbidity and mortality by means of altering cholesterol concentrations, thus leading to cardiovascular disease [40]. Unsaturated fats though have been shown to reduce cardiovascular disease risk [41]. Unsaturated fats likely reduce the risk of cardiovascular disease by decreasing the total amount of cholesterol present in the body [42]. When caloric intake from saturated fats is replaced with calories from unsaturated fat, each 5\% energy replacement is correlated with a $10 \%$ risk reduction of coronary heart disease [42].

A healthy diet alone is not entirely effective for the reduction of chronic disease as it cannot replace the beneficial effects physical activity has on the cardiovascular system [43]. Likewise, physical activity requires a healthy diet in order to maximally decrease chronic disease risk [44]. The combination of a balanced diet and exercise yield additional weight loss, along with improved metabolic risk factors including improved maximal oxygen consumption (aerobic fitness) and decreased triglycerides and blood pressure. Weight loss alone has been correlated with a decrease in certain inflammatory biomarkers [44].

\section{Clinically Prescribed Lifestyle Modifications}

Lifestyle modifications, or behavioral weight control, used 
to modify dietary habits, exercise habits, or both should be a clinician's first choice for treating obesity [30,45]. These modifications are recommended to patients with a BMI $\geq 30$ $\mathrm{kg} / \mathrm{m} 2$ or $\geq 25 \mathrm{~kg} / \mathrm{m} 2$ for those with obesity related illnesses. In prescribed lifestyle modifications, patients periodically meet with a clinician to discuss progress and help direct further lifestyle modifications as needed [45].

Physical activity modifications are typically based on recognized guidelines for recommended weekly physical activity participation. The CDC recommends a minimum of 150 minutes per week of moderate intensity activity (3.0 5.9 METs), or 75 minutes per week of vigorous activity (6.0 METs or greater), or a combination of the two in bouts of 10 minutes or more [46]. Most physical activity recommendations are typically informal advice from a clinician rather than a formal prescription. This lack of standardized activity prescription is likely due to the absence of established clinical physical activity models, like those seen with recognized dietary plans.

There appears to be a difference between simply advising patients to increase physical activity and formally prescribing it. When patients were advised to increase physical activity, they tended to actually decrease physical activity by approximately 15 minutes per week [47]. When given a formal prescription for physical activity (physician advice, educational materials, and a personalized physical activity plan that varied across patients) these patients increased their activity. Six months post-prescription, both younger ( $<50$ years) and older patients increased their physical activity by an average of approximately 30 and 65 minutes per week, respectively [47]. These results suggest that a formal prescription of physical activity can be a substantial tool for encouraging patients to increase their activity.

Dietary prescription lifestyle modifications most commonly entail reducing the amount of total food and fat consumed daily in an effort to decrease caloric intake [30]. This results in an energy imbalance. Dietary modifications that restrict the intake of a particular macronutrient or group of macronutrients are recent developments [30]. Such well known dietary modifications include the Atkins (low carbohydrate intake), Ornish (low fat intake), Zone (even macronutrient distribution), and Weight Watchers (reduced overall caloric intake) diets [48]. These diets have been shown to yield an average of approximately $3.18-3.63 \mathrm{~kg}$ of weight loss across a two month period [48].

When comparing the Atkins diet to a more clinically prevalent low calorie/low fat diet, weight loss differences are statistically indistinguishable in the long-term [49]. Yet, 35 - 50\% of study participants in both of the aforementioned diet conditions prematurely left the Foster, et al. study, citing either too little weight loss or too much difficulty sustaining the diet. The diets with the most extreme restrictions (Atkins, Ornish) resulted in the greatest discontinuation rates [49]. This is problematic as after a dietary lifestyle treatment ends, patients regain about $30 \%-35 \%$ of their weight loss within the first year and nearly all of their lost weight within five years [50].
Diet prescription efficacy appears dependent on the intensity with which the behavioral modification is promoted by the prescribing clinician. The frequency of visits to a clinician and the topics of discussion are directly related to the success of a particular diet plan. Foster et al. compared the Atkins and Ornish diets with intensive in-person behavioral treatment across two years, discovering similar, high diet efficacy so long as clinicians met regularly with their patients [51]. Weight regain occurred and increased to a greater extent the less often patients met for behavioral treatment [51]. These results suggest that specific diets are not key to producing weight loss but how active a clinician is in the patient's weight loss journey.

Obstructions to the Prescription of Lifestyle Modifications

Primary care physicians are advised to screen adults for obesity and offer comprehensive weight management plans for obese adults, yet many fail to do so [52]. Out of almost 991 million physician visits in $2015,51 \%$ of those were to primary care specialists [53]. Only 15\% of those 991 million visits included some form of diet or nutrition education or counseling. Less than $10 \%$ of all visits included exercise education or counseling and less than 3\% of total visits included weight reduction education or counseling. This is disconcerning as $61 \%$ of visits included patients with at least one chronic disease [53]. Based on this data, it is evident that primary care clinicians are missing an opportunity to prescribe appropriate lifestyle modifications to an at-risk population.

While physicians receive extensive training in the use of pharmacotherapy, the same cannot be said for prescribing lifestyle modifications [54]. The training and education a physician receives may be inadequate to make appropriate dietary and physical activity prescriptions. Over 51\% of family physicians felt inappropriately trained to prescribe weight loss plans to obese patients [55].

Over $90 \%$ of medical schools offer some nutrition education, but it is often interspersed within other science courses, instead of as an exclusive nutrition course [56]. In 2004, only $30 \%$ of U.S. medical schools required a dedicated course in nutrition. While the National Academy of Sciences (NAS) recommends 25 hours of nutrition education for medical students, only about $38 \%$ of medical schools met this recommendation. Likewise, $88 \%$ of nutrition instructors in medical schools believed more time should be dedicated to the instruction of nutrition to medical students [56].

In formal physician training, content related to appropriate physical activity is taught even less frequently than nutrition content. An average of eight hours of physical activity education is provided at U.S. medical schools [57]. Approximately $39 \%$ and $56 \%$ of medical schools do not teach aerobic and resistance training guidelines, respectively. Perhaps worse still, $56 \%$ of medical schools believe they offer sufficient physical activity education [57]. A lack of adequate physical activity and health coaching instruction leaves many practitioners underprepared to utilize this beneficial 
modality to prevent and treat chronic disease [58]. Medical students are not often exposed to lifestyle medicine, the branch of medicine that deals with espousing and sustaining healthy lifestyle behaviors.

\section{The Case for Lifestyle Medicine}

Clinicians should screen all patients for physical inactivity and poor dietary patterns. Physical activity and nutritional assessments should be treated as vital signs and patients should understand the health risks associated with a sedentary lifestyle and an unbalanced diet. Patient activity level and nutritional status are imperative to their longevity and more importantly, their quality of life. Standardizing physician assessment and prescription of physical activity and diet should be paramount. Most pharmaceutical treatments follow standardized, yet individualized practitioner prescribed schedules. Why then should the prescription of physical activity and diet protocols be any different? Standardized care provides several benefits, which include risk reduction, reduced payer costs, and consistent outcomes for different patients [59].

Several factors must be considered to program physical activity and exercise for a patient. Standardized exercise training programs could be developed for populations with common presentations (e.g. BMI ranges, specific chronic diseases, or overall morbidity and mortality risk). In many cases, evidence of effective programming for such populations and patients with specific diseases already exists. Individual dietary prescription outside of consultation with a certified dietary professional should be based on patient preference and should interfere the least with any pre-existing conditions the patient may have. Standardized treatment protocols could be established by leading experts in the field for unique patient populations. Primary care clinicians may then select the most appropriate lifestyle protocol for each patient. Clinicians could then make modifications depending on each patient's individual needs, or refer them to an exercise physiologist or dietician for further refined care as needed. An alternative approach could include primary care clinics staffed with a dietician and exercise physiologist to facilitate this patient-centered care. Patients could the visit the dietician and exercise physiologist with greater frequency than the physician promoting lifestyle change maintenance.

Primary care clinicians should prescribe physical activity and diet as a curative and preventative measure for chronic disease. Recognizing that weight loss pharmaceuticals may not reduce cardiovascular metabolic risk factors alone, a concomitant treatment with physical activity and dietary prescription (combination therapy) would likely increase weight loss and improve metabolic disease risk factors [13, 21]. For cases of extreme obesity, bariatric surgery combined with an insurance subsidized prescription diet and exercise plan may be the most viable option for a successful weight reduction, metabolic risk factor normalization, and weight loss maintenance [28].

Additional training in exercise and nutrition could be in- cluded during a medical student's education. Dedicating a course in medical school towards the prescription of lifestyle medicine for the prevention of chronic disease may positively influence physician confidence regarding the prescription of exercise and nutrition. Physician residency programs could recommend or require a rotation in lifestyle medicine for medical students interested in primary care practice or specialties that focus on patients with chronic disease. If residency programs were to favor those individuals with training in lifestyle medicine, it may provoke more interest in these preventative treatments. At the very least this would expose medical students to lifestyle treatments they may use in later practice.

In order for current primary care physicians to provide lifestyle counseling and preventive prescriptions they should be better trained in lifestyle medicine. It has been shown that even a three-hour seminar on the benefits of exercise in medicine is sufficient to increase exercise prescription by physicians by $28 \%$ [60]. While this may be enough to stimulate the prescription of lifestyle modifications it is not enough training to comprehensively address the benefits and use of physical activity for the prevention and treatment of chronic disease. In 2004, the American College of Lifestyle Medicine (ACLM) set out to define lifestyle medicine and promote its use through physician education. The ACLM advocates that all primary care physicians meet certain leadership, knowledge, assessment, management, and office and community competencies in order to effectively treat and prevent chronic disease with the practice of lifestyle medicine. Many of these competencies reflect what has been discussed in this review and include promoting healthy behavior and prescribing exercise and nutrition preventatively and curatively.

\section{Limitations to the Prescription of Lifestyle Medicine}

The major limitation to the prescription of exercise and nutrition is the heavy burden placed on the patient alone. Taking a medication is relatively easy, whereas consuming a healthy diet and exercising regularly is not. Further still, if lifestyle alterations are not sustained, excess weight regain is inevitable. These limitations make quick and simple solutions like pharmaceuticals and surgery more appealing options, despite arguably worse results and increased financial burden.

The authors acknowledge that educating physicians and changing how they prescribe exercise and nutrition is just the first step of reforming primary care to effectively combat chronic disease. Advances in the elimination of chronic disease lies mostly outside of the physician's office. Patient behavior change is paramount to the success of lifestyle medicine. Patient non-compliance with an effective prescription of exercise and nutrition is as detrimental as a lack of this prescription in the first place. A shift away from curative treatment towards preventative treatment not only requires physician education and compliance, but necessitates a rearrangement of societal values. We must encourage deviation from a culture focused on instant gratification. Exercise 
and nutritional behavior change requires prolonged effort while current culture promotes care that minimizes the energy required on the patient's end. Additionally, increased funds should be available for lifestyle medicine. Insurance providers would need to increase the accessibility to and reimbursement for lifestyle medical care.

\section{CONCLUSIONS}

Primary care clinicians are not appropriately utilizing lifestyle modifications as a preventive measure against or treatment for chronic disease. The increasing obesity prevalence in the United States is a strong indicator of a fundamentally flawed approach to health care. This obesity epidemic is directly related to elevated rates of mortality resulting from chronic disease. Current pharmaceutical and surgical treatments provide minimal relief to the obesity and chronic disease epidemic. Physical activity and balanced nutrition are paramount to the effective treatment of obesity and chronic disease. Primary care clinicians should increase their familiarity with exercise programming and dietary protocols to better provide comprehensive preventive and curative lifestyle modifications. In order to effectively combat chronic disease, clinicians should underscore the necessity of lifestyle treatment and prioritize its use in clinical settings. If primary care medical providers continue to neglect lifestyle medicine, lifespan and quality of life will be negatively impacted. Despite revolutionary medical advances in pharmacology and surgery over the past few centuries, there is no substitute for the benefits realized with appropriate physical activity and a balanced diet.

\section{ACKNOWLEDGEMENTS}

The authors would like to thank the participants and the undergraduate students who helped in data collection for this work.

\section{Conflicts of Interest}

The authors declare no conflict of interest.

\section{REFERENCES}

1. Bauer UE, Briss PA, Goodman RA, Bowman BA. Prevention of chronic disease in the 21st century: elimination of the leading preventable causes of premature death and disability in the USA. Lancet. 2014; 384(9937):45-52.

2. Fryar CD, Carroll MD, Ogden CL. Prevalence of overweight, obesity, and severe obesity among adults aged 20 and over: United States, 1960-1962 through 20152016. National Center for Health Statistics. 2018; https:// www.cdc.gov/nchs/data/hestat/obesity_adult_15_16/ obesity_adult_15_16.htm. (Accessed Feb 20, 2018)

3. Gerteis J, Izrael D, Deitz D, et al. Multiple chronic con- ditions chart book. Rockville (MD): Agency for Healthcare Research and Quality; 2014. Secondary. 2014.

4. Heron MP. National Vital Statistics Reports: Deaths: Leading causes for 2016. National Center for Health Statistics. 2018; https://www.cdc.gov/nchs/nvss/deaths. htm.

5. WHO. Global status report on noncommunicable diseases 2010. 2011; https://www.who.int/nmh/publications/ncd_report2010/en/. (Accessed Feb 26, 2018)

6. WHO. Global status report on noncommunicable diseases 2014. 2014; https://www.who.int/nmh/publications/ncd-status-report-2014/en/. (Accessed Feb 26, 2018)

7. Healy GN, Dunstan DW, Salmon J, et al. Objectively measured light-intensity physical activity is independently associated with 2-hr plasma glucose. Diabetes care. 2007.

8. Owen N, Bauman A, Brown W. Too much sitting: a novel and important predictor of chronic disease risk? Br J Sports Med. 2009; 43(2):81-83.

9. Ekelund U, Steene-Johannessen J, Brown WJ, et al. Does physical activity attenuate, or even eliminate, the detrimental association of sitting time with mortality? A harmonised meta-analysis of data from more than 1 million men and women. Lancet. 2016; 388(10051):1302-1310.

10. O'Rahilly S, Farooqi IS. Human Obesity: A Heritable Neurobehavioral Disorder That Is Highly Sensitive to Environmental Conditions. Diabetes. 2008; 57(11):2905-2910.

11. Hales CM, Carroll MD, Fryar CD, Ogden CL. NCHS Data Brief: Prevalence of Obesity Among Adults and Youth: United States, 2015-2016. 2017; https:// www.cdc.gov/nchs/products/databriefs/db288.htm. (Accessed March 10, 2018)

12. Erlandson M, Ivey LC, Seikel K. Update on Office-Based Strategies for the Management of Obesity. Am Fam Physician. 2016; 94(5):361-368.

13. Yanovski SZ, Yanovski JA. Long-term drug treatment for obesity: a systematic and clinical review. JAMA. 2014; 311(1):74-86.

14. Bogers RP, Bemelmans WJ, Hoogenveen RT, et al. Association of overweight with increased risk of coronary heart disease partly independent of blood pressure and cholesterol levels: a meta-analysis of 21 cohort studies including more than 300000 persons. Arch Intern Med. 2007; 167(16):1720-1728.

15. Hampp C, Kang EM, Borders-Hemphill V. Use of prescription antiobesity drugs in the United States. Pharmacotherapy. 2013; 33(12):1299-1307.

16. Kuhn M, Campillos M, Letunic I, Jensen LJ, Bork P. A side effect resource to capture phenotypic effects of drugs. Mol Syst Biol. 2010; 6(1):343.

17. Krentz AJ, Fujioka K, Hompesch M. Evolution of pharmacological obesity treatments: focus on adverse side-effect profiles. Diabetes Obes Metab. 2016; 18(6):558-570.

18. Onakpoya IJ, Heneghan CJ, Aronson JK. Post-mar- 
keting withdrawal of anti-obesity medicinal products because of adverse drug reactions: a systematic review. BMC Med. 2016; 14(1):191.

19. Perret-Guillaume C, Joly L, Benetos A. Heart rate as a risk factor for cardiovascular disease. Prog Cardiovasc Dis. 2009; 52(1):6-10.

20. Dong Z, Xu L, Liu H, Lv Y, Zheng Q, Li L. Comparative efficacy of five long-term weight loss drugs: quantitative information for medication guidelines. Obes Rev. 2017; 18(12):1377-1385.

21. Manning S, Pucci A, Finer N. Pharmacotherapy for obesity: novel agents and paradigms. Ther Adv Chronic Dis. 2014; 5(3):135-148.

22. Fruhbeck G. Bariatric and metabolic surgery: a shift in eligibility and success criteria. Nat Rev Endocrinol. 2015; 11(8):465-477.

23. Chang SH, Stoll CR, Song J, Varela JE, Eagon CJ, Colditz GA. The effectiveness and risks of bariatric surgery: an updated systematic review and meta-analysis, 20032012. JAMA Surg. 2014; 149(3):275-287.

24. English WJ, DeMaria EJ, Brethauer SA, Mattar SG, Rosenthal RJ, Morton JM. American Society for Metabolic and Bariatric Surgery estimation of metabolic and bariatric procedures performed in the United States in 2016. Surg Obes Relat Dis. 2018; 14(3):259-263.

25. Mechanick JI, Youdim A, Jones DB, et al. Clinical practice guidelines for the perioperative nutritional, metabolic, and nonsurgical support of the bariatric surgery patient-2013 update: cosponsored by American Association of Clinical Endocrinologists, the Obesity Society, and American Society for Metabolic \& Bariatric Surgery. Obesity. 2013; 21(S1):S1-S27.

26. Dindo D, Demartines N, Clavien PA. Classification of surgical complications: a new proposal with evaluation in a cohort of 6336 patients and results of a survey. Ann Surg. 2004; 240(2):205-213.

27. Omalu BI, Ives DG, Buhari AM, et al. Death rates and causes of death after bariatric surgery for Pennsylvania residents, 1995 to 2004. Arch Surg. 2007; 142(10):923928.

28. Zalesin KC, Franklin BA, Miller WM, et al. Preventing weight regain after bariatric surgery: an overview of lifestyle and psychosocial modulators. Am J Lifestyle Med. 2010; 4(2):113-120.

29. Sjostrom L, Lindroos AK, Peltonen M, et al. Lifestyle, diabetes, and cardiovascular risk factors 10 years after bariatric surgery. N Engl J Med. 2004; 351(26):26832693.

30. Wadden TA, Webb VL, Moran CH, Bailer BA. Lifestyle modification for obesity: new developments in diet, physical activity, and behavior therapy. Circulation. 2012; 125(9):1157-1170.

31. Willis LH, Slentz CA, Bateman LA, et al. Effects of aerobic and/or resistance training on body mass and fat mass in overweight or obese adults. J Appl Physiol. 2012; 113(12):1831-1837.

32. Salvadori A, Fanari P, Marzullo P, et al. Short bouts of anaerobic exercise increase non-esterified fatty acids release in obesity. Eur J Nutr. 2014; 53(1):243-249.

33. Pedersen BK, Febbraio MA. Muscles, exercise and obesity: skeletal muscle as a secretory organ. Nat Rev Endocrinol. 2012; 8(8):457.

34. McAuley PA, Chen H, Lee DC, Artero EG, Bluemke DA, Burke GL. Physical activity, measures of obesity, and cardiometabolic risk: the Multi-Ethnic Study of Atherosclerosis (MESA). J Phys Act Health. 2014; 11(4):831-837.

35. Bei Y, Zhou Q, Sun Q, Xiao J. Exercise as a platform for pharmacotherapy development in cardiac diseases. Curr Pharm Des. 2015; 21(30):4409-4416.

36. Lee DC, Artero EG, Sui X, Blair SN. Mortality trends in the general population: the importance of cardiorespiratory fitness. J Psychopharmacol. 2010; 24(S4):27-35.

37. WHO. The world health report 2002 - reducing risks, promoting healthy life. 2002; http://www.who.int/ whr/2002/en/.

38. HHS and USDA. 2015 - 2020 Dietary Guidelines for Americans. 8th Edition. 2015; https://health.gov/dietaryguidelines/2015/guidelines/. (Accessed Mar 29, 2018)

39. Slavin JL, Lloyd B. Health benefits of fruits and vegetables. Adv Nutr. 2012; 3(4):506-516.

40. German JB, Dillard CJ. Saturated fats: what dietary intake? Am J Clin Nutr. 2004; 80(3):550-559.

41. Mozaffarian D, Micha R, Wallace S. Effects on coronary heart disease of increasing polyunsaturated fat in place of saturated fat: a systematic review and meta-analysis of randomized controlled trials. PLoS Med. 2010; 7(3):e1000252.

42. Jones PJ, Lichtenstein AH, Schaefer EJ, Namchuk GL. Effect of dietary fat selection on plasma cholesterol synthesis in older, moderately hypercholesterolemic humans. Arterioscler Thromb. 1994; 14(4):542-548.

43. Andersson A, Bryngelsson S. Towards a healthy diet: from nutrition recommendations to dietary advice. Food Nutr Res. 2007; 51(1):31-40.

44. Schwingshackl L, Dias S, Hoffmann G. Impact of longterm lifestyle programmes on weight loss and cardiovascular risk factors in overweight/obese participants: a systematic review and network meta-analysis. Syst Rev. 2014; 3(1):130.

45. Butryn ML, Webb V, Wadden TA. Behavioral treatment of obesity. Psychiatr Clin North Am. 2011; 34(4):841859.

46. HHS. 2008 Physical Activity Guidelines for Americans. 2008; https://health.gov/paguidelines/2008/. (Accessed Apr 4, 2018)

47. Grandes G, Sanchez A, Sanchez-Pinilla RO, et al. Effectiveness of physical activity advice and prescription by physicians in routine primary care: a cluster randomized trial. Arch Intern Med. 2009; 169(7):694-701.

48. Dansinger ML, Gleason JA, Griffith JL, Selker HP, Schaefer EJ. Comparison of the Atkins, Ornish, Weight Watchers, and Zone diets for weight loss and heart dis- 
ease risk reduction: a randomized trial. JAMA. 2005; 293(1):43-53.

49. Foster GD, Wyatt HR, Hill JO, et al. A randomized trial of a low-carbohydrate diet for obesity. N Engl J Med. 2003; 348(21):2082-2090.

50. Blomain ES, Dirhan DA, Valentino MA, Kim GW, Waldman SA. Mechanisms of Weight Regain following Weight Loss. ISRN Obes. 2013; 2013:210524.

51. Foster GD, Wyatt HR, Hill JO, et al. Weight and metabolic outcomes after 2 years on a low-carbohydrate versus low-fat diet: a randomized trial. Ann Intern Med. 2010; 153(3):147-157.

52. Kraschnewski JL, Sciamanna CN, Stuckey HL, et al. A silent response to the obesity epidemic: decline in US physician weight counseling. Med Care. 2013; 51(2):186-192.

53. Rui P, Okeyode T. National Ambulatory Medical Care Survey: 2015 State and National Summary Tables. 2015; https://www.cdc.gov/nchs/ahcd/web_tables.htm. (Accessed Apr 12, 2018)

54. Persson G, Brorsson A, Ekvall Hansson E, Troein M, Strandberg EL. Physical activity on prescription (PAP) from the general practitioner's perspective - a qualitative study. BMC Fam Pract. 2013; 14(1):128.

55. Foster GD, Wadden TA, Makris AP, et al. Primary care physicians' attitudes about obesity and its treatment. Obes Res. 2003; 11(10):1168-1177.

56. Adams KM, Lindell KC, Kohlmeier M, Zeisel SH. Status of nutrition education in medical schools. Am J Clin Nutr. 2006; 83(4):S941-S944.

57. Stoutenberg M, Stasi S, Stamatakis E, et al. Physical activity training in US medical schools: Preparing future physicians to engage in primary prevention. Phys Sportsmed. 2015; 43(4):388-394.

58. Clarke CA, Hauser ME. Lifestyle Medicine: A Primary Care Perspective. J Grad Med Educ. 2016; 8(5):665-667.

59. Mannion R, Exworthy M. (Re) Making the procrustean bed? Standardization and customization as competing logics in healthcare. Int J Health Policy Manag. 2017; 6(6):301-304.

60. Windt J, Windt A, Davis J, Petrella R, Khan K. Can a 3-hour educational workshop and the provision of practical tools encourage family physicians to prescribe physical activity as medicine? A pre-post study. BMJ. 2015; 5(7):e007920. 